\title{
Pneumococcal pneumonia presenting with septic shock: host- and pathogen-related factors and outcomes
}

\author{
C Garcia-Vidal, ${ }^{1}$ C Ardanuy, ${ }^{2}$ F Tubau, ${ }^{2}$ D Viasus, ${ }^{1}$ J Dorca, ${ }^{3}$ J Liñares, ${ }^{2}$ F Gudiol, ${ }^{1}$ \\ J Carratalà ${ }^{1}$
}

${ }^{1}$ Infectious Disease Department of Hospital Universitari de Bellvitge, Institut d'Investigació Biomèdica de Bellvitge (IDIBELL), Spanish Network for the Research in Infectious Diseases (REIPI), University of Barcelona, L'Hospitalet de Llobregat, Barcelona, Spain;

${ }^{2}$ Microbiology Department of Hospital Universitari de Bellvitge, Institut d'Investigació Biomèdica de Bellvitge (IDIBELL), Ciber de Enfermedades Respiratorias (CIBERES), University of

Barcelona, L'Hospitalet de Llobregat, Barcelona, Spain;

${ }^{3}$ Respiratory Department of Hospital Universitari de Bellvitge, Institut d'Investigació Biomèdica de Bellvitge (IDIBELL), University of Barcelona, L'Hospitalet de Llobregat, Barcelona, Spain

Correspondence to: Dr J Carratalà, Infectious Disease Department, Hospital Universitari de Bellvitge, Feixa Llarga s/n, 08907 L'Hospitalet de Llobregat, Barcelona, Spain jcarratala@ub.edu

Received 14 July 2009 Accepted 14 October 2009 Published Online First 2 December 2009

\section{ABSTRACT}

Background: Host- and pathogen-related factors associated with septic shock in pneumococcal pneumonia are not well defined. The aim of this study was to identify risk factors for septic shock and to ascertain patient outcomes. Serotypes, genotypes and antibiotic resistance of isolated strains were also analysed.

Methods: Observational analysis of a prospective cohort of non-severely immunosuppressed hospitalised adults with pneumococcal pneumonia. Septic shock was defined as a systolic blood pressure of $<90 \mathrm{~mm} \mathrm{Hg}$ and peripheral hypoperfusion with the need for vasopressors for $>4 \mathrm{~h}$ after fluid replacement.

Results: 1041 patients with pneumococcal pneumonia diagnosed by Gram stain and culture of appropriate samples and/or urine antigen test were documented, of whom 114 (10.9\%) had septic shock at admission. After adjustment, independent risk factors for shock were current tobacco smoking $(\mathrm{OR}, 2.11 ; 95 \% \mathrm{Cl}, 1.02$ to 4.34 ; $p=0.044)$, chronic corticosteroid treatment $(O R, 4.45$; $95 \% \mathrm{Cl}, 1.75$ to $11.32 ; \mathrm{p}=0.002)$ and serotype 3 (OR, 2.24; $95 \% \mathrm{Cl}, 1.12$ to $4.475 ; \mathrm{p}=0.022$ ). No significant differences were found in genotypes and rates of antibiotic resistance. Compared with the remaining patients, patients with septic shock required mechanical ventilation more frequently ( $37 \%$ vs $4 \% ; p<0.001)$ and had longer length of stay (11 vs 8 days; $p<0.001$ ). The early $(10 \%$ vs $1 \% ; p<0.001)$ and overall case fatality rates $(25 \%$ vs $5 \% ; p<0.001)$ were higher in patients with shock.

Conclusions: Septic shock is a frequent complication of pneumococcal pneumonia and causes high morbidity and mortality. Current tobacco smoking, chronic corticosteroid treatment and infection caused by serotype 3 are independent risk factors for this complication.

Streptococcus pneumoniae remains a major cause of disease worldwide. ${ }^{1}$ Among pneumonia pathogens, it is the most common cause of hospitalisation in adults and the most frequent cause of death. ${ }^{23}$ Despite continuing improvements in aetiological diagnosis, effective antibiotic treatment and advances in supportive care, mortality rates in patients with pneumococcal pneumonia remain high, ranging from $5 \%$ to $35 \%{ }^{4}$

Septic shock at admission is one of the most important factors influencing prognosis of patients with pneumococcal pneumonia. It is known that the intrinsic relationship between the host and the microorganism is critical in the pathogenesis of septic shock. ${ }^{5}$ Many host factors influence the ability to develop an optimal immune response against infection. Moreover, $>90$ serotypes of pneumococci have been described, defined by differences in the polysaccharide capsule, ${ }^{6}$ the major virulence determinant of these organisms. Improvement in the knowledge of serotypes associated with septic shock may help in the design of new vaccines. However, within serotypes, different genotypes may be identified ${ }^{8-11}$ and these genotypes are associated with the presence of different virulence determinants. The association between infecting $S$ pneumoniae genotype and pneumonia severity has not been well established.

To date, little information is available regarding the host- and pathogen-related factors associated with pneumococcal pneumonia presenting with septic shock. We performed this prospective study to identify risk factors for septic shock and to ascertain clinical outcomes in a large and homogeneous cohort of non-severely immunosuppressed hospitalised adults with pneumococcal pneumonia. Serotypes, genotypes and antibiotic resistance of isolated strains were also analysed.

\section{METHODS}

\section{Setting, patients and study design}

The study was carried out in a 900-bed university hospital for adults in Barcelona, Spain. The hospital serves an area of 1000000 inhabitants and admits $\sim 24000$ patients per year. All nonseverely immunosuppressed adults admitted to the hospital with pneumococcal pneumonia through the emergency department from 13 February 1995 to 30 June 2008 were prospectively recruited and followed-up. Patients with neutropenia, HIV infection and transplantation were not included.

For the purposes of this study patients were divided into two groups: patients who presented with septic shock at admission and those who did not. (henceforth "pneumococcal pneumonia presenting with shock" and "pneumococcal pneumonia without shock"

\section{Clinical evaluation and follow-up}

At the initial visit, before starting empirical antibiotic treatment, patients underwent a complete clinical history and physical examination. Microbiological studies included two sets of blood cultures and sputum Gram stain and culture when available. Urinary antigen detection for $S$ pneumoniae was performed if indicated by the attending doctor. Patients were stratified into risk classes by using the validated prediction rule calculated 
according to the Pneumonia Severity Index (PSI) scores, as described elsewhere. ${ }^{12}$

Empirical antibiotic treatment was administered according to hospital guidelines, which recommend the administration of a $\beta$-lactam agent (ceftriaxone or amoxicillin-clavulanate) with or without a macrolide or a fluoroquinolone. Combination treatment was recommended for patients with clinical suspicion of Legionella or an atypical pathogen or in the case of severe pneumonia in the absence of a demonstrative sputum Gram stain. Levofloxacin monotherapy was allowed for selected cases (ie, patients with allergy to $\beta$-lactam agents and no prior quinolone use).

Patients were seen daily during their hospital stay by one or more of the investigators who provided medical advice when requested and recorded clinical and microbiological data in a computer-assisted protocol.

\section{Definitions}

Pneumococcal pneumonia was diagnosed in patients with signs and symptoms of an acute-onset lower respiratory tract infection, a new infiltrate on chest radiograph, one or more cultures positive for $S$ pneumoniae obtained from blood, normally sterile fluids or sputum, and/or a positive test for detection of urinary antigen. Only good quality samples of sputum $(<10$ squamous epithelial cells and $>25$ leucocytes per field) were accepted for processing.

The diagnosis of septic shock was based on a systolic blood pressure of $<90 \mathrm{~mm} \mathrm{Hg}$ and peripheral hypoperfusion with the need for vasopressors for $>4 \mathrm{~h}$ after fluid replacement at admission. Current tobacco smoking was recorded when a patient had smoked $>10$ cigarettes per day for at least 1 year preceding the study, and alcohol consumption when a patient had consumed $>80 \mathrm{~g}$ of alcohol daily for at least 1 year preceding the study. Chronic corticosteroid treatment was defined as $\leqslant 20 \mathrm{mg} /$ day of prednisone or its equivalent for a period of at least 30 days; patients receiving higher doses were excluded. A patient was considered to have been pneumococcal vaccinated if 23 -valent polysaccharide pneumococcal vaccine had been administered in the 5 years before admission, according to the hospital and primary healthcare centre records. A patient was considered influenza vaccinated if influenza vaccine had been administered during the year prior to admission.

Early case fatality rate was defined as death due to any cause $\leqslant 48 \mathrm{~h}$ after hospitalisation. Overall case fatality rate was defined as death due to any cause during $\leqslant 30$ days of hospitalisation.

\section{Microbiological studies}

$S$ pneumoniae was identified using standard microbiology procedures. From 2000 onwards, urinary antigen detection using a rapid immunochromatographic assay (Now, Binax, Portland, Maine, USA) for $S$ pneumoniae was also available. Antimicrobial susceptibility was tested by microdilution method, following the Clinical Laboratory Standard Institute methods and criteria. ${ }^{13}{ }^{14}$

Serotype was identified using the Quellung reaction at the Spanish Reference Laboratory ${ }^{15}$ and/or by PCR following the methodology described by the Centers for Disease Control (CDC; http://www.cdc.gov/ncidod/biotech/strep/strepindex. $\mathrm{htm})$. Unusual serotype-genotype combinations were retested.

For molecular typing, all available strains were typed using pulsed-field gel electrophoresis (PFGE) after restriction with
SmaI. PFGE patterns were compared with those of representative international pneumococcal clones of the Pneumococcal Molecular Epidemiology Network. ${ }^{16}$ Sixty-six selected strains representative of main clusters (at least one strain for each PFGE pattern accounting for $>2$ pneumococci) were studied using multilocus sequence typing, as described elsewhere. ${ }^{11}$ Allele numbers and sequence types (STs) were assigned using the pneumococcal multilocus sequence typing Web site.

\section{Statistical analysis}

To detect significant differences between groups, we used the $\chi^{2}$ or Fisher exact test for categorical variables, and the Student $t$ test or the Mann-Whitney $U$ test for continuous variables, when appropriate. The multivariate analysis of factors potentially associated with septic shock at admission included all the significant variables in the univariate analysis with clinical relevance. We used the stepwise logistic regression model of the SPSS software package (SPSS, version 13.5; SPSS, Chicago, Illinois, USA). Statistical significance was established at $\alpha=0.05$. All reported $p$ values are two tailed.

\section{RESULTS}

Over the study period, 1041 consecutive adults with pneumococcal pneumonia were included, of whom 114 (10.9\%) had septic shock at admission. Table 1 details the microbiological methods used to establish the diagnosis of pneumococcal pneumonia.

Clinical features of the patients according to the study group (pneumococcal pneumonia presenting with shock and pneumococcal pneumonia without shock) are shown in table 2 . Patients with shock were significantly younger, were more frequently current smokers, had more frequently received chronic corticosteroid treatment and were less likely to have received prior influenza vaccine than patients without shock. They also had more frequent respiratory failure, multilobar pneumonia and impaired consciousness at admission and were more commonly classified into high-risk PSI classes than those without.

Antibiotic-resistant pneumococci with a penicillin minimum inhibitory concentration (MIC) $\geqslant 4 \mu \mathrm{g} / \mathrm{ml}(1.5 \%$ vs $1.7 \% ; \mathrm{p}=1)$

Table 1 Microbiological diagnosis of pneumococcal pneumonia

\begin{tabular}{|c|c|c|}
\hline & $\begin{array}{l}\text { Pneumococcal } \\
\text { pneumonia } \\
\text { presenting with } \\
\text { shock }\end{array}$ & $\begin{array}{l}\text { Pneumococcal } \\
\text { pneumonia without } \\
\text { shock }\end{array}$ \\
\hline & $\mathrm{n}=114(\%)$ & $\mathrm{n}=927(\%)$ \\
\hline One positive test & $77(67.5)$ & 731 (78.8) \\
\hline Gram stain and sputum culture & $12(10.5)$ & $234(25.2)$ \\
\hline Blood cultures & $15(13.2)$ & $109(11.8)$ \\
\hline Pleural fluid culture & $4(3.5)$ & $36(3.9)$ \\
\hline $\begin{array}{l}\text { Transthoracic needle aspiration } \\
\text { specimen culture }\end{array}$ & $1(0.9)$ & $6(0.6)$ \\
\hline Urinary antigen test & $45(39.5)$ & $346(37.3)$ \\
\hline More than one positive test & $37(32.5)$ & $196(21.1)$ \\
\hline $\begin{array}{l}\text { Gram stain and sputum culture, } \\
\text { blood cultures and urinary antigen } \\
\text { test }\end{array}$ & $6(5.3)$ & $13(1.4)$ \\
\hline $\begin{array}{l}\text { Gram stain and sputum culture, } \\
\text { and blood cultures }\end{array}$ & $7(6.1)$ & $55(5.9)$ \\
\hline $\begin{array}{l}\text { Blood cultures and urinary antigen } \\
\text { test }\end{array}$ & $19(16.7)$ & $98(10.6)$ \\
\hline $\begin{array}{l}\text { Gram stain and sputum culture, } \\
\text { and urinary antigen test }\end{array}$ & $5(4.4)$ & $30(3.2)$ \\
\hline
\end{tabular}


Table 2 Patients' demographic and clinical characteristics

\begin{tabular}{|c|c|c|c|}
\hline \multirow[b]{2}{*}{ Characteristic } & $\begin{array}{l}\text { Pneumococcal } \\
\text { pneumonia } \\
\text { presenting with } \\
\text { shock }(n=114) \\
\end{array}$ & $\begin{array}{l}\text { Pneumococcal } \\
\text { pneumonia } \\
\text { without shock } \\
(\mathrm{n}=927) \\
\end{array}$ & \multirow[b]{2}{*}{ p Value } \\
\hline & No. (\%) & No. (\%) & \\
\hline \multicolumn{4}{|l|}{ Demographics } \\
\hline Median age (range) & $64(18-92)$ & $70(18-100)$ & 0.003 \\
\hline Age $\geqslant 70$ & $34(29.8)$ & $459(49.5)$ & $<0.001$ \\
\hline Male, sex & $67(58.8)$ & $618(66.7)$ & 0.093 \\
\hline \multicolumn{4}{|l|}{ Vaccination status } \\
\hline Influenza vaccine (season) & 25 (21.9) & $403(43.5)$ & 0.007 \\
\hline Pneumococcal vaccination & $12(10.5)$ & $134(14.5)$ & 0.874 \\
\hline Current tobacco abuse & $48(42.1)$ & $254(27.4)$ & 0.001 \\
\hline Heavy alcohol consumption & $23(20.2)$ & $195(21.0)$ & 0.831 \\
\hline Underlying disease & $84(73.7)$ & $742(80.0)$ & 0.109 \\
\hline COPD & $33(28.9)$ & $271(29.2)$ & 1.00 \\
\hline Diabetes mellitus & $21(18.4)$ & $187(20.2)$ & 0.711 \\
\hline Cancer & $11(9.6)$ & $82(8.8)$ & 0.730 \\
\hline Cerebrovascular disease & $6(5.3)$ & $49(5.3)$ & 1.00 \\
\hline Chronic heart disease & $34(29.8)$ & $211(22.8)$ & 0.238 \\
\hline Chronic renal disease & $7(6.0)$ & $55(5.9)$ & 0.836 \\
\hline Chronic liver disease & $14(12.3)$ & $78(8.4)$ & 0.165 \\
\hline Dementia & $2(1.8)$ & $28(3.0)$ & 0.764 \\
\hline Previous antibiotic treatment & $17(14.9)$ & $166(17.9)$ & 0.786 \\
\hline $\begin{array}{l}\text { Chronic corticosteroid treatment } \\
\text { ( } \leqslant 20 \mathrm{mg} / \text { day) }\end{array}$ & $14(12.3)$ & $55(5.9)$ & 0.015 \\
\hline \multicolumn{4}{|l|}{$\begin{array}{l}\text { Clinical characteristics at } \\
\text { admission }\end{array}$} \\
\hline Median temperature (range) & $37.5(35.0-41.0)$ & $38.0(35.5-40.0)$ & $<0.001$ \\
\hline Median respiratory rate (range) & $32(11-55)$ & $28(12-50)$ & $<0.001$ \\
\hline Respiratory failure & $84(73.7)$ & $538(58.0)$ & 0.002 \\
\hline Multilobar pneumonia & 47 (41.2) & $198(21.4)$ & $<0.001$ \\
\hline Pleural effusion & $27(23.7)$ & $194(20.9)$ & 0.746 \\
\hline Impaired consciousness & $35(30.7)$ & $114(12.3)$ & $<0.001$ \\
\hline $\begin{array}{l}\text { High-risk pneumonia (PSI }>90 \\
\text { points, classes IV and V) }\end{array}$ & $91(79.8)$ & $552(59.5)$ & $<0.001$ \\
\hline
\end{tabular}

COPD, chronic obstructive pulmonary disease; PSI, Pneumonia Severity Index.

and a cefotaxime MIC of $1 \mu \mathrm{g} / \mathrm{ml}(0 \%$ vs $1.9 \%$; $\mathrm{p}=0.617)$ were distributed equally between groups. No differences in the rates of resistance to erythromycin ( $11.8 \%$ vs $16.4 \% ; p=0.384)$, chloramphenicol ( $1.4 \%$ vs $4.2 \% ; p=0.502)$, tetracycline $(8.8 \%$ vs $8.1 \% ; p=0.938)$ and cotrimoxazole $(28.3 \%$ vs $28.5 \%$; $p=0.908)$ isolates were found between groups.

Five hundred and ninety-eight $(92.0 \%)$ pneumococci were available for serotyping and 474 (73.1\%) for molecular typing. The most frequent serotypes are summarised in table 3 . Serotype 3 was the most frequent serotype causing pneumococcal pneumonia and was the most commonly associated with septic shock. Conversely, serotype 1 was rarely associated with this complication. The most common genotypes were ST260 (22.6\% vs $15.2 \%$, respectively), Spain ${ }^{9 \mathrm{~V}}$ ST156 (7.5\% vs $11.2 \%$ ), Netherlands $^{3}$ ST180 (11.3\% vs 7.8\%), Netherlands ${ }^{7 \mathrm{~F}}$ ST191 (7.5\% vs $4 \%$ ) and Netherlands ${ }^{8}$ ST53 (9.4\% vs $\left.4.5 \%\right)$. Two sequence types were found among serotype 3 : the predominant one was ST260 ${ }^{3}$ and the second most predominant was ST180 (identifier of Netherlands ${ }^{3}$ ST180 PMEN clone). No significant differences were found between the groups regarding genotypes.

The antibiotic treatment and clinical outcomes by study groups are shown in table 4. No differences were found regarding the median time to institution of initial antibiotic treatment. Bacteraemia occurred more frequently in patients with shock. This group also required mechanical ventilation more frequently, and had longer length of hospital stay.
Moreover, the early and overall case fatality rates were higher in patients who had septic shock at admission.

Table 5 summarises the results of the multivariate analysis of factors potentially associated with septic shock. After adjustment, independent risk factors were current tobacco smoking, chronic corticosteroid treatment and infection caused by $S$ pneumoniae serotype 3 . In a subanalysis of the 322 episodes of bacteraemic pneumococcal pneumonia, the only independent risk factor for septic shock was the use of chronic corticosteroid treatment (OR 6.087, 95\% CI 1.903 to 19.469).

\section{DISCUSSION}

This prospective study offers a comprehensive evaluation of the host- and pathogen-related risk factors associated with septic shock in pneumococcal pneumonia. We found that $10.9 \%$ of patients had septic shock at admission, a figure similar to that encountered in previous studies of community-acquired pneumonia, in which the incidence of septic shock ranged from $7.4 \%$ to $10 \%{ }^{12}{ }^{17}$

In our study, independent factors for septic shock were current tobacco smoking, chronic corticosteroid treatment and infection caused by $S$ pneumoniae serotype 3 . Neither infecting genotype nor antibiotic resistance was found to be associated with septic shock.

Tobacco smoking has been previously associated with increased risk of infection, ${ }^{18-21}$ especially invasive pneumococcal disease. ${ }^{22}$ It has been demonstrated that smoking negatively affects the immune system, compromising the host's ability to mount an appropriate immune and inflammatory response..$^{23-25}$ To our knowledge, our study is the first to link current tobacco smoking directly with septic shock in pneumococcal pneumonia. This finding provides additional support to encourage patients at risk for pneumonia to undergo smoking cessation programmes.

Glucocorticoids exert a decisive influence on the immune function of macrophages and granulocytes, as well as in systemic cytokine expression. Additionally, chronic corticosteroid treatment suppresses the production of corticotrophinreleasing hormone and corticotrophin, and can induce adrenal atrophy ${ }^{26}$ Consequently, the adrenal responses to infection may be insufficient to control the inflammatory situation. Although evidence from randomised trials is lacking, treatment with supplementary corticosteroids may be of benefit in patients

Table 3 Serotype distribution of 650 Streptococcus pneumoniae isolates

\begin{tabular}{lllll}
\hline & $\begin{array}{l}\text { Pneumococcal } \\
\text { pneumonia } \\
\text { presenting with } \\
\text { shock (n= 69) }\end{array}$ & & $\begin{array}{l}\text { Pneumococcal } \\
\text { pneumonia without } \\
\text { shock (n= 581) }\end{array}$ & \\
\cline { 2 - 2 } & No. (\%) & & No. (\%) & p Value \\
\hline Available serotype & $65(94.2)$ & & $533(91.7)$ & 0.475 \\
3 & $25(38.5)$ & & $127(23.8)$ & 0.010 \\
8 & $5(7.7)$ & & $26(4.9)$ & 0.367 \\
7 & $5(7.7)$ & $22(4.1)$ & 0.200 \\
$19 \mathrm{~F}$ & $4(6.2)$ & $23(4.3)$ & 0.521 \\
$19 \mathrm{~A}$ & $4(6.2)$ & & $22(4.1)$ & 0.512 \\
4 & $4(6.2)$ & & $25(4.7)$ & 0.543 \\
1 & $1(1.5)$ & & $53(9.9)$ & 0.025 \\
14 & $3(4.6)$ & $28(5.3)$ & 1.00 \\
$9 \mathrm{~V}$ & $2(3.1)$ & $24(4.5)$ & 1.00 \\
$23 \mathrm{~F}$ & $1(1.5)$ & $13(2.4)$ & 1.00 \\
\hline
\end{tabular}


Table 4 Antibiotic treatment and clinical outcomes by study group

\begin{tabular}{|c|c|c|c|}
\hline \multirow[b]{2}{*}{ Treatment and outcomes } & \multirow{2}{*}{$\begin{array}{l}\text { Pneumococcal } \\
\text { pneumonia } \\
\text { presenting with } \\
\text { shock (n= 114) } \\
\text { No. }(\%) \\
\end{array}$} & \multirow{2}{*}{$\begin{array}{l}\begin{array}{l}\text { Pneumococcal } \\
\text { pneumonia without } \\
\text { shock }(\mathrm{n}=927)\end{array} \\
\text { No. }(\%)\end{array}$} & \multirow[b]{2}{*}{ p Value } \\
\hline & & & \\
\hline \multicolumn{4}{|l|}{ Initial antibiotic treatment } \\
\hline$\beta$-Lactam monotherapy & $52(45.6)$ & $566(61.1)$ & 0.002 \\
\hline $\begin{array}{l}\beta \text {-Lactam + macrolide or } \\
\text { fluoroquinolone }\end{array}$ & $55(48.2)$ & $246(26.5)$ & $<0.001$ \\
\hline Levofloxacin & $6(5.2)$ & $100(10.8)$ & 0.071 \\
\hline Other & $1(0.8)$ & $15(1.6)$ & 1.00 \\
\hline $\begin{array}{l}\text { Door-to-needle antibiotic } \\
\text { time in hours, median } \\
\text { (range) }\end{array}$ & $5.00(1-20)$ & $5.00(1-22)$ & 0.057 \\
\hline Bacteraemia & $47(41.2)$ & $275(29.7)$ & 0.014 \\
\hline $\begin{array}{l}\text { Intensive care unit } \\
\text { admission }\end{array}$ & $55(48.2)$ & $64(6.9)$ & $<0.001$ \\
\hline $\begin{array}{l}\text { Need for mechanical } \\
\text { ventilation }\end{array}$ & $42(36.8)$ & $41(4.4)$ & $<0.001$ \\
\hline $\begin{array}{l}\text { Length of hospital stay in } \\
\text { days, median (range) }\end{array}$ & $11(1-89)$ & $8(1-97)$ & $<0.001$ \\
\hline Early case fatality rate & $11(9.6)$ & $8(0.9)$ & $<0.001$ \\
\hline Overall case fatality rate & $29(25.4)$ & $48(5.2)$ & $<0.001$ \\
\hline
\end{tabular}

with pneumococcal pneumonia and septic shock in whom there is evidence of adrenal insufficiency.

Serotype 3 is usually associated with adult pneumococcal infection and is less frequent in children and in young adults. In our study it caused nearly one in four of the cases of pneumococcal pneumonia. Interestingly, we found that serotype 3 was independently associated with septic shock. Previous studies associated serotype 3 with a low invasive potential but with higher mortality rates in bacteraemic pneumococcal pneumonia. ${ }^{27-33}$ Probably, within serotype 3 isolates, the genetic background plays an important role in virulence. In our study, two main genotypes were found among serotype 3 strains (ST260 and ST180) and no significant differences were observed among patients with or without shock at presentation.

The 23-valent polysaccharide vaccine includes serotype 3 and is recommended for use in adults in many countries. However, vaccination rates among target persons remain low. The current 7-valent paediatric conjugate pneumococcal vaccine (PCV) lacks serotype 3 . It has been previously demonstrated that the introduction of PCV7 has changed the serotypes causing invasive pneumococcal disease in adults. ${ }^{1}$ Importantly, the forthcoming 13-valent PCV includes this serotype and might help to prevent septic shock in pneumococcal pneumonia. Moreover, in the future the PCV13 vaccine will be available for adult vaccination.

It is known that influenza virus alters the lungs in a way that predisposes to adherence, invasion and induction of disease by pneumococcus. ${ }^{34}$ It has been documented that prior influenza vaccination prevents pneumonia hospitalisations and has a substantial effect on all-cause mortality. ${ }^{34}$ In our study, although influenza vaccination was found to be a protective factor for septic shock in the univariate analysis, this finding did not reach significance in the final model.

We found that patients presenting with septic shock required mechanical ventilation more frequently and had a longer length of hospital stay than patients without shock. As expected, these patients had high early and overall case fatality rates. These findings corroborate those of previous studies. ${ }^{12} 3536$
Table 5 Risk factors for septic shock at admission for pneumococcal pneumonia by multivariate analysis

\begin{tabular}{lll}
\hline & OR (95\% CI) & p Value \\
\hline Age $\geqslant 70$ & $0.70(0.32$ to 1.54$)$ & 0.376 \\
Current tobacco smoking & $2.11(1.02$ to 4.34$)$ & 0.044 \\
Chronic corticosteroid treatment & $4.45(1.75$ to 11.32$)$ & 0.002 \\
Prior influenza vaccine (season) & $0.55(0.26$ to 1.21$)$ & 0.138 \\
Serotype 3 & $2.24(1.12$ to 4.475$)$ & 0.022 \\
Serotype 1 & $0.26(0.03$ to 1.97$)$ & 0.190 \\
Bacteraemia & $1.50(0.75$ to 2.99$)$ & 0.251 \\
\hline
\end{tabular}

Despite a number of strengths, our study has some limitations that should be acknowledged. First, the possible role of polymorphisms of innate immunity was not analysed. Investigating the relationship between immune system genetics and the severity of infection should therefore be a major focus of future research. Secondly, the small number of cases of pneumonia caused by some serotypes and genotypes precluded analysing their potential correlation with shock. Thirdly, the study was carried out in a single centre and the results might not translate to other geographic areas.

In summary, septic shock is a frequent complication of pneumococcal pneumonia and causes high morbidity and mortality. Current tobacco smoking, chronic corticosteroid treatment and infection caused by $S$ pneumoniae serotype 3 are independent risk factors for this serious complication.

Funding: This study was supported by research grant REIPI RD06/0008 from the Ministerio de Sanidad y Consumo, Instituto de Salud Carlos III, Spanish Network for the Research in Infectious Diseases; CIBER de Enfermedades Respiratorias (CIBERES; CB06/06/0037), which is a project run by the ISCIII (Instituto de Salud Carlos III), Madrid, Spain; Fondo de Investigación Sanitaria de la Seguridad Social (grants 04/ 0139, 07/0864 and PI060647); and by Institut d'Investigació Biomèdica de Bellvitge (CG-V). We acknowledge the use of the Streptococcus pneumoniae MLST web site at Imperial College London, which is funded by the Wellcome Trust. We are grateful to M. Alegre for her excellent technical support.

Competing interests: None.

Ethics approval: The study was approved by the Ethics Committee of theHospital Universitari de Bellvitge.

Provenance and peer review: Not commissioned; externally peer reviewed.

\section{REFERENCES}

1. Ardanuy C, Tubau F, Pallarés R, et al. Epidemiology of invasive pneumococcal disease among adult patients in Barcelona before and after pediatric 7-valent pneumococcal conjugate vaccine introduction 1997-2007. Clin Infect Dis 2009:48:57-64.

2. Shariatzadeh MR, Huang JQ, Tyrrell GJ, et al. Bacteremic pneumococcal pneumonia: a prospective study in Edmonton and neighboring municipalities. Medicine (Baltimore) 2005;84:147-61.

3. Rosón B, Carratalà J, Dorca J, et al. Etiology, reasons for hospitalization, risk classes and outcomes of patients with community-acquired pneumonia hospitalized on the basis of conventional admission criteria. Clin Infect Dis 2001;33:158-65.

4. Feikin DR, Schuchat A, Kolczak M. Mortality from invasive pneumococcal pneumonia in the era of antibiotic resistance, 1995-1997. Am J Public Health 2000;90:223-9.

5. Van Der Poll T, Opal S. Host-pathogen interactions in sepsis. Lancet Infect Dis 2008;8:32-43.

6. Henrichsen J. Six newly recognized types of Streptococcus pneumoniae. J Clin Microbiol 1995; 33:2759-62.

7. Kamerling JP. Pneumococcal polysaccharides: a chemical view. In: Tomasz A, ed. Streptococcus pneumoniae, molecular biology and mechanisms of disease. New York: Mary Ann Liebert, 2000:81-114.

8. Muller-Graf CDM, Whatmore AM, King SJ, et al. Population biology of Streptococcus pneumoniae isolated from oropharyngeal carriage and invasive disease. Microbiology 1999;145:3283-93.

9. Enright MC, Spratt BG. Multilocus sequence typing. Trends Microbiol 1999;7:482-7.

10. Llull D, Lopez R, Garcia E. Clonal origin of the type 37 Streptococcus pneumoniae. Microb Drug Resist 2000;6:269-75.

11. Enright MC, Spratt BG. A multilocus sequence typing scheme for Streptococcus pneumoniae: identification of clones associated with serious invasive disease. Microbiology 1998;144:3049-60. 
12. Fine MJ, Auble TE, Yealy DM, et al. A prediction rule to identify low-risk patients with community-acquired pneumonia. N Engl J Med 1997;336:243-50.

13. Clinical Laboratory Standard Institute (CLSI). Performance standards for antimicrobial susceptibility testing: 19th informational supplement. CLSI document M100-S18. Wayne, PA: CLSI, 2009.

14. Clinical and Laboratory Standard Institute (CLSI). Methods for dilution antimicrobial susceptibility test for bacteria that growth aerobically; approved standard: 7th edn. CLSI documentM7-A6. Wayne, PA: CLSI, 2006.

15. Fenoll A, Granizo JJ, Aguilar $L$, et al. Temporal trends of invasive Streptococcus pneumoniae serotypes and antimicrobial resistance patterns in Spain from 1979 to 2007. J Clin Microbiol 2009:47:1012-20.

16. McGee L, McDougal L, Zhou J, et al. Nomenclature of major antimicrobial-resistant clones of Streptococcus pneumoniae defined by the pneumococcal molecular epidemiology network. J Clin Microbiol 2001;39:2565-71.

17. Mortensen E, Coley C, Singer D, et al. Causes of death for patients with communityacquired pneumonia: results from the Pneumonia Patient Outcomes Research Team Cohort Study. Arch Intern Med 2002;162:1059-64.

18. Kark JD, Lebiush M, Rannon L. Cigarette smoking as a risk factor for epidemic a(h1n1) influenza in young men. N Engl J Med 1982;307:1042-6.

19. Bates Mn, Khalakdina A, Pai M, et al. Risk of tuberculosis from exposure to tobacco smoke: a systematic review and meta-analysis. Arch Intern Med 2007;167:335-42.

20. Hirschtick RE, Glassroth J, Jordan MC, et al. Bacterial pneumonia in persons infected with the human immunodeficiency virus. N Engl J Med 1995;333:845-51.

21. Straus WL, Plouffe JF, File TM Jr, et al. Risk factors for domestic acquisition of legionnaires' disease. Arch Intern Med 1996:156:1685-92.

22. Nuorti JP, Butler JC, Farley MM, et al. Cigarette smoking and invasive pneumococcal disease. Active Bacterial Core Surveillance Team. N Engl J Med 2000; 342:681-9.

23. Chen H, Cowan M, Hasday J, et al. Tobacco smoking inhibits expression of proinflammatory cytokines and activation of IL-1R-associated kinase, p38, and NF-kB in alveolar macrophages stimulated with TLR2 and TLR4 agonists. J Immunol 2007:179:6097-106.

24. Sopori M. Effects of cigarette smoke on the immune system. Nat Rev Immunol 2002;2:372-7.

25. McCrea KA, Ensor JE, Nall K, et al. Altered cytokine regulation in the lungs of cigarette smokers. Am J Respir Crit Care Med 1994;150:696-703.

26. Cooper M, Stewart P. Corticosteroid insufficiency in acutely ill patients. N Engl J Med 2003; $348: 727-34$

27. Austrian R, Gold J. Pneumococcal bacteremia with special reference to bacteremic pneumococcal pneumonia. Ann Intern Med 1964:60:759-70.

28. Henriques B, Kalin M, Ortqvist A, et al. Molecular epidemiology of Streptococcus pneumoniae causing invasive disease in five countries. J Infect Dis 2000;182:833-9.

29. Finland M, Barnes MW. Changes in occurrence of capsular serotypes of Streptococcus pneumoniae at Boston City Hospital during selected years between 1935 and 1974. J Clin Microbiol 1977:5:154-66.

30. Gransden WR, Eykyn SJ, Phillips I. Pneumococcal bacteraemia: 325 episodes diagnosed at St. Thomas's Hospital. BMJ 1985;290:505-8.

31. Martens $\mathbf{P}$, Worm SW, Lundgren B, et al Serotype-specific mortality from invasive Streptococcus pneumoniae disease revisited. BMC Infect Dis 2004;4:21

32. Jansen A, Rodenburg G, Van der Ende A, et al. Invasive pneumococcal disease among adults: associations among serotypes, disease characteristics, and outcome. Clin Infect Dis 2009; 49:e23-29.

33. Alanee SRJ, McGee L, Jackson D, et al. Association of serotypes of Streptococcus pneumoniae with disease severity and outcome in adults: an international study. Clin Infect Dis 2007;45:46-51.

34. McCullers JA. Insights into the interaction between influenza virus and pneumococcus. Clin Microbiol Rev 2006;19:571-82.

35. Garcia-Vidal C, Fernández-Sabé N, Carratalà J, et al. Early mortality in patients with community-acquired pneumonia: causes and risk factors. Eur Respir $J$ 2008:32:733-9

36. Fine MJ, Smith MA, Carson CA, et al. Prognosis and outcomes of patients with community-acquired pneumonia. A meta-analysis. JAMA 1996:275:134-41. 